\title{
Resistance to extinction of a running response after a small number of partially rewarded trials'
}

C. THOMAS SURRIDGE, MICHAEL E. RASHOTTE AND ABRAM AMSEL

UNIVERSITY OF TORONTO

Data are presented which indicate that, under our experimental conditions, the partial reinforcement extinction effect does not occur after only one NRNR sequence of trials. This finding appears to hold whether or not the partially rewarded trials are preceded by a number of continuously rewarded trials.

An explanation of the partial reinforcement effect (PRE) in terms of frustrative nonreward attributes the PRE "to the evocation of frustration by nonreward in partial reinforcement, to the conditioning of anticipatory frustration $\left(\mathrm{r}_{F^{-S}}\right)$ to cues in the instrumental sequence, and to the association of $\mathrm{S}_{\mathrm{F}}$ with approach responses in acquisition" (Amsel, 1962, p. 311). According to this interpretation, nonreward does not become frustrating until sufficient anticipatory reward $\left(r_{R}\right)$ has developed: thus, the conditioning of $s_{F}$ to approach would seem to depend on some minimal number of partially rewarded training trials.

Several experiments have been published recently demonstrating the PRE after a sequence of only three or four PRF trials including two nonrewards (e.g., McCain, 1965a, 1965b, 1966; McCain, Love, \& Gruer, 1962). The McCain experiments included a period of preliminary adjustment, prior to runway training, which could conceivably result in the build up of $r_{R}$ in relation to cues which later are present in the training situation. That is, in some experiments Ss were allowed to approach and eat wet mash after leaving $E$ 's hand, in others Ss were fed large amounts of wet mash a short time after being in the runway to be used in training, and in most experiments pretraining involved both of these procedures. In the present study, we eliminated any possibility of such factors in the first experiment, but allowed them to operate in the second experiment in the form of a set number of rewarded training trials.

The questions this paper attempts to answer are, then, twofold. First, will a PRE occur following a small number of PRF trials when no preliminary feeding related to handling or exploration of the alley is allowed? Second, will a PRE be evident if the small number of PRF trials are preceded by a number of CRF trials?

\section{Method}

Experiment 1. Forty-eight male albino rats, approximately 60-70 days of age, were maintained on a 23-hr. 10-gm food deprivation schedule for 21 days prior to experimental training, during which time they were handled and tamed. Ss were not fed in relation to handling and were not allowed to explore the experimental apparatus.

A straight, black, 5-ft. 3-in. wooden runway with a gray entry box and start box was employed. Running time was measured from the opening of the start door to the breaking of a photobeam 3 in. from a food cup attached to the end wall, providing a performance measure over $5 \mathrm{ft}$. of the alley.

Ss were randomly assigned to one of three groups. Group $C$ received four successive rewarded acquisition trials; Group P, two rewards (R) and two nonrewards (N) in an NRNR sequence; Group $N$, four successive nonrewarded "acquisition" trials. All Ss were run a total of 14 trials, four acquisition trials followed by 10 extinction trials, with an intertrial interval (ITI) of 20-25 sec. Reward was two 500-mg Noyes pellets. Ss were detained in the goal box for $90 \mathrm{sec}$. on every trial.

Experiment II. Twenty male albino rats approximately 90 days old were maintained on a $23-\mathrm{hr} .10-\mathrm{gm}$ food deprivation schedule for about 30 days prior to experimental training during which time they were handled and tamed. There was no feeding in relation to handling and no exploration of the apparatus was allowed.

A 5-ft. 3-in. straight black runway with a gray start box was employed in this experiment. Ss were not handled at the start of a trial, but were maintained in the experimental room in individual boxes which served as entry boxes to the runway. Running time was measured from the opening of the start door to the breaking of a photobeam 3 in. from the end wall, providing a performance measure over the first $5 \mathrm{ft}$. of the alley.

Ss were randomly assigned to one of two groups which were run four trials a day, with a 25-30 min. ITI and one 250-mg Noyes pellet as reward, Days 1-6 were CRF for both groups. On day 7 Group $C$ received four CRF trials while Group P received an NRNR sequence of PRF trials. Both groups were extinguished on days 8-15.

\section{Results and Discussion}

The time data from each experiment were converted to speed scores (ft./sec.) and are presented in the two panels of Fig. 1.

In Experiment I acquisition performance of Group $P$ remained inferior to that of Group $C$, there being a tendency for higher speeds after Trials 2 and 4 (re- 


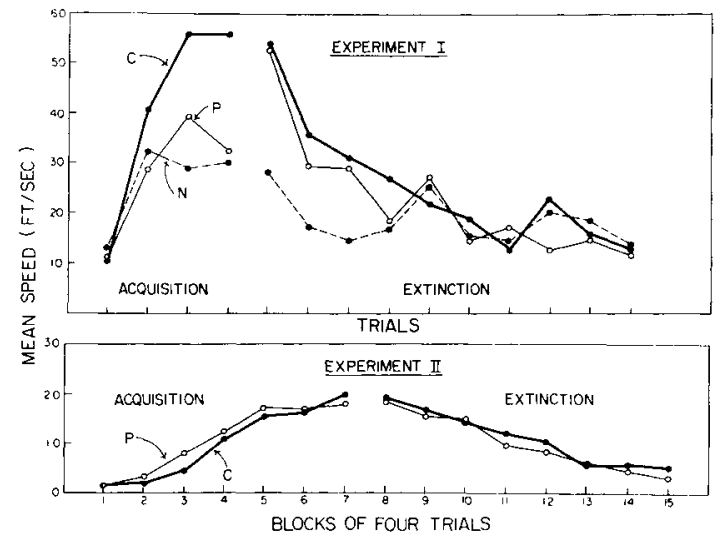

Fig. 1. Acquisition and extinction speed data for Experiments I and II.

warded trials) and lower speeds after Trial 3 (nonrewarded trial). By the first extinction trial, which in fact represents terminal acquisition performance, Groups $\mathrm{P}$ and $\mathrm{C}$ were running at about the same speed. Although for the next few trials performance of Group $P$ appeared somewhat inferior to that of Group $C$, analysis of variance comparing Groups $P$ and $C$ over the first four extinction trials yielded only a significant Trials effect $(p<.001)$. The Group main effect and the Group by Trials interaction were not significant $(p>$ $.05)$. Group $N$ (never rewarded) showed an increase and then a decrease in speed over the course of the experiment and did not reach the level of Groups $\mathrm{C}$ or $P$ at the end of acquisition, i.e., Trial 5 .

In Experiment II acquisition speeds of Group C were somewhat lower than those of Group P for all except the last block of trials. An analysis of variance of these data yielded a nonsignificant Group effect, a significant Blocks effect $(p<.001)$, and a Group by Block interaction which barely reached significance at the .05 level, $F=2.51$, df $=6 / 108$ and which presumably reflects sampling error. The two groups appeared to extinguish at approximately the same rate, with the overall speed
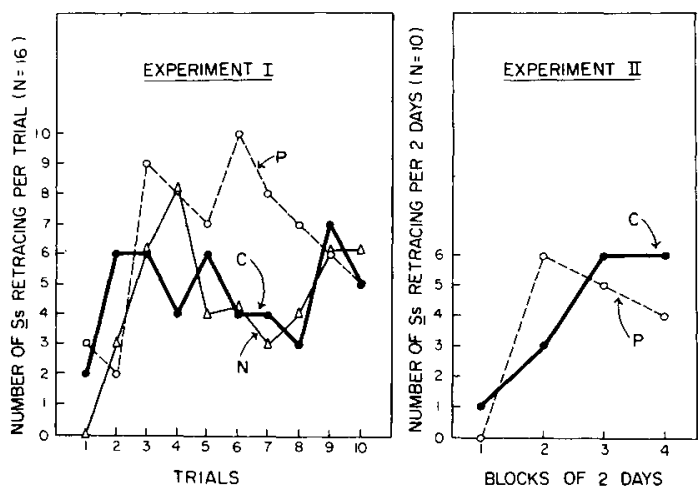

Fig. 2. Extinction retrace data for Experiments I and II. of Group P being somewhat less than Group C. Analysis of variance over the eight extinction days yielded nonsignificant Group and Group by Blocks effects, while the Block main effect was significant, $F=43.84$, $\mathrm{df}=$ $7 / 128, \mathrm{p}<.001$.

A record was kept in both experiments of all extinction trials on which $\mathrm{S}$ retraced (turned completely around) in the runway. These are presented in Fig. 2 as the number of Ss retracing per trial (Experiment I), and the number of Ss retracing per 2-day block (Experiment II).

In Experiment I more Ss retraced in Group $P$ than in Group C. A t test comparing the mean number of retraces for these groups indicated that the difference was reliable, $t=2.75, d f=31, p<.01$ for a one-tailed test. Group $N$ showed an operant retrace baseline and did not differ from Group C. In Experiment in Groups $\mathrm{P}$ and $\mathrm{C}$ showed no systematic differences in retracing.

In summary, there was no indication in Experiment I of a conventional PRE but, if anything, a suggestion of faster extinction after PRF relative to CRF acquisition. The data of Experiment II would seem to indicate that even where the four PRF trials are preceded by 24 rewards, there is no evidence of a PRE relative to an appropriate CRF comparison group, and these data fail to support our explanation of McCain's results in terms of the build-up of anticipatory reward by extensive pre-experimental training.

Finally, our data suggest that great caution should be exercised before extinction differences after very small numbers of acquisition trials are taken as representing a "true" PRE: by some criteria (see Anderson, 1963) Group $\mathrm{N}$ of Experiment I, which was never rewarded in the experiment, shows the greatest resistance to extinction in terms of speed (Fig. 1) and is more resistant to extinction than Group $P$ in terms of retracing (Fig. 2).

\section{References}

Amsel, A. Frustrative nonreward in partial reinforcement and discrimination learning: Some recent history and a theoretical extension. Psychol. Rev., 1962, 69, 306-328.

Anderson, N. H. Comparison of different populations: Resistance to extinction and transfer. Psychol. Rev., 1963, 70, 162-179.

McCain, G. Partial reinforcement with a small number of acquisition trials: Pretraining and acquisition. Psychon. Sci., 1965a, 2, 249-250.

McCain, G. Partial reinforcement with a small number of trials: Performance in extinction. Psychon. Sci., 1965b, 2, 131-132. McCain, G. Partial reinforcement effects following a small number of acquisition trials. Psychon. Monogr. Suppl., 1966, 1, 12. McCain, G., Love, N., \& Gruer, W. Extinction as a function of a small number of partially reinforced trials. Psychol. Rep., 1962, $11,451-454$.

\section{Note}

1. Supported by research grants GB-3772 from the National Science Foundation and $A P B-72$ from the National Research Council of Canada. 